Revista Internacional de Ciencias Podológicas

ISSN: 1989-5151

http://dx.doi.org/10.5209/RICP.59828

\title{
Revisión bibliográfica sobre la técnica quirúrgica de fenol-alcohol en el tratamiento de la onicocriptosis
}

\author{
Gibert Perelló, Loreto'; Sánchez Gómez, Rubén²
}

Fecha de recepción: 24 de enero de 2018 / Fecha de aceptación: 2 de abril de 2018

\section{Resumen.}

La onicocriptosis es una patología ungueal común que suele afectar al primer dedo del pie, que cursa con dolor y limitación funcional, siendo la técnica de fenol-alcohol una de las más usadas, por sus buenos resultados funcionales y estéticos.

El objetivo del presente estudio fue realizar una revisión bibliográfica sobre esta técnica quirúrgica para el tratamiento de la onicocriptosis, para plasmar la mejora en las diferentes variables estudiadas por los autores.

Labúsqueda bibliográfica se realizó en el portal científico Medline ${ }^{\circledR}$ (Pubmed) usando los descriptores “onychocryptosis”, "phenol", "phenol alcohol" y "phenol matricectomy alcohol". Después de la aplicación de los filtros seleccionados, se seleccionaron 14 artículos para hacer la revisión.

Tras analizar las variables, podemos destacar que hay una prevalencia notoria en el uso de fenol-alcohol frente a fenol; el tabaco pueden alterar el tiempo de cicatrización y el uso de antibióticos enmascarar los signos de la infección. Además con la buena praxis de la técnica, se observan bajas tasas de recurrencia y el grado de dolor es de baja/media intensidad, lo que favorece una pronta incorporación a las actividades de la vida diaria.

Palabras clave: técnica fenol; fenol-alcohol; onicocriptosis; matricectomía

\section{[en] Review about phenol-alcohol technique surgery on onychocryptosis treatment.}

\begin{abstract}
.
Onychocryptosis is a common ungueal disease that usually affects to hallux, giving pain and functional limitation, being phenol-alcohol matricectomy the technique more used for these pathology thanks to it functional and static good results.

The aim of this study was to make a review about this technique to show the improvement into different variables research for the author's bibliography. The search was made through Medline ${ }^{\circledR}$ scientific gate (pubmed) with descriptors "onychocryptosis", "phenol", "phenol alcohol" and "phenol matricectomy alcohol".

After different filters application, 14 papers it was selected to make the review, and the analysis variables showed that there was a high prevalence of phenol-alcohol technique over phenol technique isolated; tobacco use can modify cicatrization times and antibiotics use can mask infections signs; a good praxis diminish recurrence and pain postsurgery, improving the quickly incorporation to normal live.
\end{abstract}

Key words: phenol technique; phenol-alcohol technique; onychocryptosis; matricectomy

Los autores declaran no tener ningún tipo de interés económico o comercial.

Sumario: 1. Introducción. 1.1. Definición; epidemiología. 1.2. Clínica. 1.3. Tratamiento. 2. Material y métodos. 3. Discusión. 3.1. Tiempo de cicatrización. 3.2. El dolor postquirúrgico. 3.3. El exudado de la herida postquirúrgica. 3.4. Infección. 3.5. Conclusiones. 4. Bibliografía.

\footnotetext{
Diplomada en Podología. Clínica Pododinámica. Madrid. España

loretogibert@gmail.com

2 Profesor asociado. Universidad Complutense de Madrid. España.

rusago_@hotmail.com

Paseo de las Acacias n61, local bajo A

28005, Madrid
} 
Cómo citar: Gibert Perelló, L.; Sánchez Gómez, R. (2018) Revisión bibliográfica sobre la técnica quirúrgica de fenolalcohol en el tratamiento de la onicocriptosis, en Revista internacional de ciencias podológicas 12(2), 99-107.

\section{Introducción}

\subsection{Definición; epidemiología}

La onicocriptosis es una afección de la lámina ungueal en la que se daña el pliegue ungueal. Es una patología común que produce dolor, inflamación y limitación funcional. Afecta principalmente al primer dedo (hallux) y en menor medida al resto de dedos ${ }^{1}$.

La onicocriptosis fue definida en 1845 por Lewis Durlacher como "uña que crece hacia el interior de la carne". Esta definición puede resultar confusa ya que el ancho de la uña depende del ancho de la matriz, no se ensancha cuando se da la patología ${ }^{2,3}$. Pearson et $\mathrm{al}^{4}$ argumentaron sin embargo que en la mayoría de los casos la forma de la uña no era la causante de la onicocriptosis, sino que era debida a una inflamación del rodete medial de la uña que impedía la zona de su crecimiento normal.

En cuanto a la epidemiología, los resultados consultados en la bibliografía varían según autor y estudio. Algunos autores como Khan et $\mathrm{al}^{5}$. sostienen que la onicocriptosis afecta $69 \%$ hombres frente a $31 \%$ mujeres, al igual que Bryant et $\mathrm{al}^{6}$, que afirman que afecta a hombres más que a mujeres, sobre todo entre los 1425 años aunque pero puede darse a cualquier edad, de la misma manera que en un estudio estadístico en cirugía ungueal de la Escuela de Podología de Barcelona ${ }^{7}$, en el que se indicaba que prevalecían los hombres $(58 \%)$ frente a las mujeres (41.58\%), o Martínez Nova et $\mathrm{al}^{1}$ quién fijó en un $62 \%$ a los hombres y en un $38 \%$ en mujeres. Frente a esto, encontramos autores como Carmona y Fernández- Morato ${ }^{8}$ que refirieron que la onicocriptosis es más frecuente en las mujeres (59\%) que en los hombres (41\%) (menos de los 9-21 años y 8083 años, que prevalecen los hombres), que se da entre la primera y la segunda décadas de la vida y que en mayores de 30 años se igualan las frecuencias, produciéndose una asociación con otras patologías tales como onicogrifosis y onicomicosis. Connelley ${ }^{9}$ y Weaber $^{10}$ relacionaron la aparición de onicocriptosis en el tratamiento con antifúngicos. La onicocriptosis se localiza principalmente en el primer dedo del pie ${ }^{11}$, y el lado más afectado es el peroneal frente al tibial $(2: 1)^{1,3,7}$.

\subsection{Clínica}

Las características clínicas de la onicocriptosis atañen a diferentes alteraciones que afectan tanto al rodete como a la placa ungueal, siendo la hipertrofia de los bordes, la presencia de infección y el dolor ${ }^{1,12}$, los principales indicadores de gravedad que marcan los grados de evolución de la patología y que presentamos a modo de resumen en la siguiente tabla: 


\begin{tabular}{|c|c|}
\hline ESTADÍO & CARACTERÍSTICAS \\
\hline I & $\begin{array}{l}\text { Eritema, edema leve, dolor a la presión en pliegue ungueal o rodete } \\
\text { lateral. } \\
\text { El pliegue ungueal es menor a } 3 \mathrm{~mm} \text { y no sobrepasa los límites de la } \\
\text { lámina. Tratamiento: conservador. }\end{array}$ \\
\hline \multirow[t]{2}{*}{$\begin{array}{l}\text { II (subdividido } \\
\text { en IIa y IIb) }\end{array}$} & $\begin{array}{l}\text { IIa: exacerbación de los síntomas del estadío I (dolor acentuado, edema } \\
\text { e hiperestesia). Puede haber drenaje seroso e infección. Pliegue ungueal } \\
<3 \mathrm{~mm} \text {. } \\
\text { Tratamiento: conservador y/o matricectomía. }\end{array}$ \\
\hline & $\begin{array}{l}\text { IIb: síntomas parecidos a IIa. Pliegue ungueal igual o mayor a } 3 \mathrm{~mm} \text {. } \\
\text { Tratamiento: matricectomía y resección de bordes hipertróficos. }\end{array}$ \\
\hline III & $\begin{array}{l}\text { Aumentan los síntomas de la etapa II, aparece tejido de granulación e } \\
\text { hipertrofia crónica del pliegue ungueal, el tejido de granulación cubre } \\
\text { la lámina ungueal. Tratamiento: reducción de tejido hipertrófico y } \\
\text { matricectomía. }\end{array}$ \\
\hline IV & $\begin{array}{l}\text { Deformidad crónica y grave de la uña, ambos rodetes periungueales y } \\
\text { rodete distal afectados. } \\
\text { Tratamiento: reducción de tejido hipertrófico y matricectomía }\end{array}$ \\
\hline
\end{tabular}

Figura 1.- Clasificación de los estadíos de la onicocriptosis.

Se aprecian los 4 estadíos de la onicocriptosis descritos por los autores, donde la hipertrofia del rodete y la inflamación /infección, son las principales variables clasificatorias.

\subsection{Tratamiento}

El objetivo de elección de la mejor técnica quirúrgica va encaminada a una recuperación correcta en la que el dolor y la funcionalidad se vean mínimamente afectados, una pronta vuelta a las actividades de la vida diaria, así como baja tasa de recurrencia ${ }^{1}$

La cirugía ungueal por técnica de fenol alcohol fue descrita en 1945 por Boll y modificadas posteriormente por diversos autores $^{8}$. Esta técnica ha sido utilizada en pacientes diabéticos controlados sin riesgo vascular y glucemias controladas ${ }^{1,8,13}$. Carmona ${ }^{8}$ en su revisión, afirma que el paciente diabético puede tolerar la quemadura química producida por el fenol. Las indicaciones para el tratamiento de la onicocriptosis incluyen dolor moderado, paroniquias de repetición, onicocriptosis crónica e infección ${ }^{11}$, afectación funcional, bordes hipertróficos, nula respuesta a tratamientos conservadores y patología ósea subyacente ${ }^{8}$.

En pos de mejorar el conocimiento a cerca de los efectos que tiene la técnica fenol-alcohol, el objetivo del presente trabajo será analizar las siguientes variables más comunes de los estudios consultados dentro de la técnica de fenol alcohol y fenol: tasa de curación, dolor, exudado de la herida postquirúrgica, recurrencia, infección y complicaciones (formación de espículas de inclusión y onicocriptosis).

\section{Material y métodos}

Para la realización de este estudio se obtuvieron las variables anteriormente descritas mediante los datos cuantitativos de las diferentes investigaciones empíricas publicadas, en la base de datos bibliográfica Medline ${ }^{\circledR}$ (Pubmed) usando los descriptores "onychocryptosis", "phenol", "phenol alcohol" y "phenol matricectomy alcohol". Fueron incluidos todos aquellos artículos en inglés o castellano, que relacionaban fenol/ fenol alcohol $y$ onicocriptosis, causante de la afección anteriormente expuesta. Además, fueron excluidos aquellos artículos que, cumpliendo los criterios de inclusión, no pudieron ser obtenidos a texto completo o estuvieran repetidos en otras bases de datos.

Tras esta búsqueda, se obtuvieron 124 resultados cuando se unieron los descriptores "onychocryptosis AND phenol", 19 artículoscon los descriptores "onychocryptosis AND phenol 
alcohol" y 8 artículos con "onychocryptosis AND phenol matricectomy alcohol".

Los filtros de búsqueda de los tipos de estudio que nos interesaban para llevar a cabo el análisis fueron los siguientes: Meta-Analysis, Randomized Controlled Trial, Review, y Systematic Reviews, quedando 41 artículos para el descriptor "onychocryptosis AND phenol" de los cuales, 30 de ellos hablaban de fenol alcohol, 3 artículos para el descriptor "onychocryptosis AND phenol alcohol" y sin artículos para el descriptor "onychocryptosis AND phenol matricectomy alcohol". Tras realizar lectura crítica se descartaron 39 artículos por no ajustarse a nuestros criterios de búsqueda sobre variables o sobre el tipo de artículo buscado. Para obtener información sobre las variables tasa de curación, dolor, exudado de la herida postquirúrgica, recurrencia, infección y formación de espículas de inclusión, utilizamos un total de 9 artículos (3 fenol ${ }^{14,15,18}$ y 6 de fenol-alcohol ${ }^{5,13,1617,19,20}$ ), y para la discusión se añadieron 4 artículos, de los cuales 3 hablaban sobre fenol alcohol $25,26,27$ y uno de ellos sobre profilaxis antibiótica en cirugía de onicocriptosis ${ }^{24}$. La ecuación de búsqueda se expone de manera detallada en la figura 2. El diagrama de flujo de la depuración de artículos, aparece en la figura 3.

\begin{tabular}{|l|l|}
\hline Bases de datos & Ecuaciones de búsqueda \\
\hline MEDLINE (www.pubmed.com) & $\begin{array}{l}\text { ((NCBI website "Onychocryptosis) AND (MH phenol) } \\
\text { AND (MH phenol alcohol) AND (MH matricentomy } \\
\text { phenol alcohol)) OR (MH alcohols) OR (MH phenols)). }\end{array}$ \\
\hline
\end{tabular}

Figura 2. Ecuaciones de búsqueda

Abreviaturas: NCBI (National Center for Biotechnology information), $\mathrm{MH}$ (terminus de búsqueda MeSH).

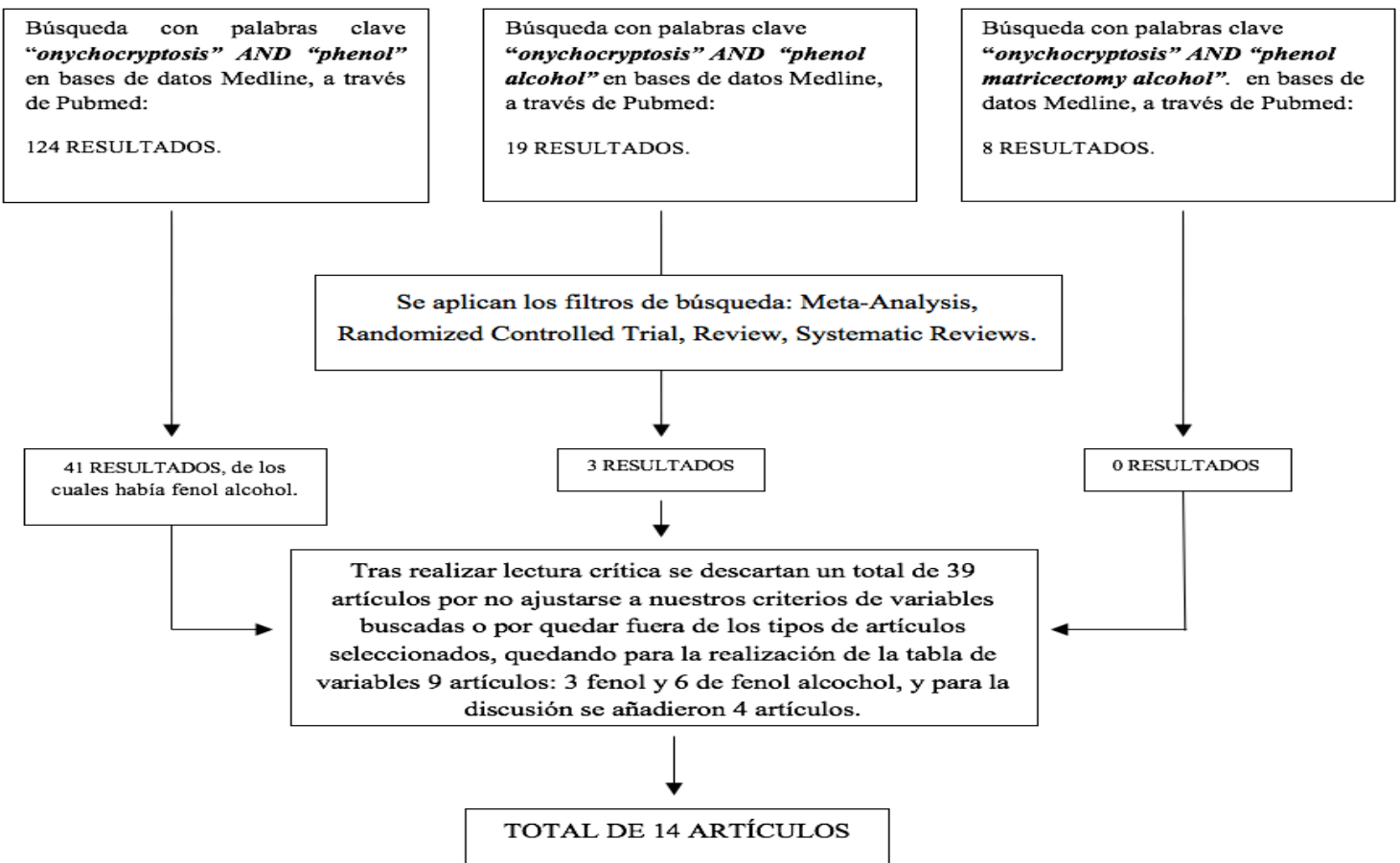

Figura 3: diagrama de flujo.

Depuración de los artículos seleccionados hasta llegar a la muestra final de 14 resultados.

Elaboración propia

A continuación se muestra una tabla en

la que se exponen las diferentes variables analizadas de los estudios seleccionados: 


\begin{tabular}{|c|c|c|c|c|}
\hline$\stackrel{\mathscr{E}}{0}$ & 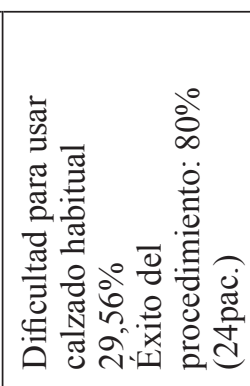 & & & 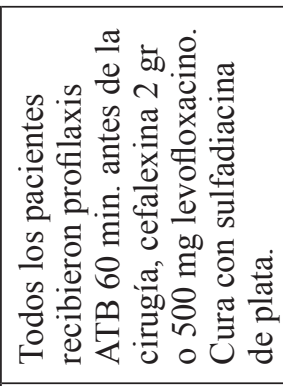 \\
\hline 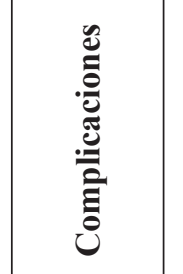 & $\stackrel{\circ}{z}$ & & 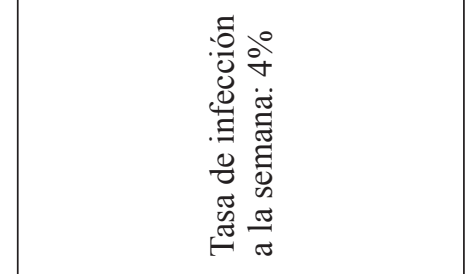 & \\
\hline 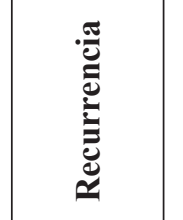 & 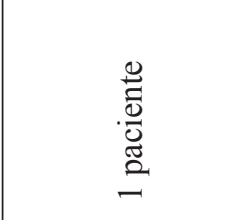 & 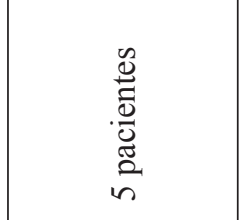 & $\stackrel{\circ}{\circ}$ & \\
\hline 宸 & 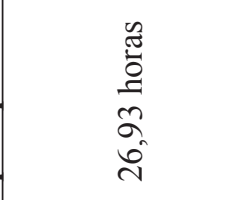 & & & \\
\hline 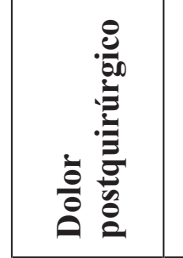 & 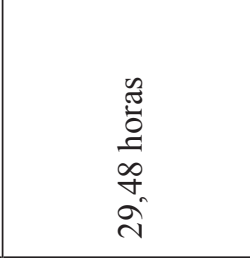 & & 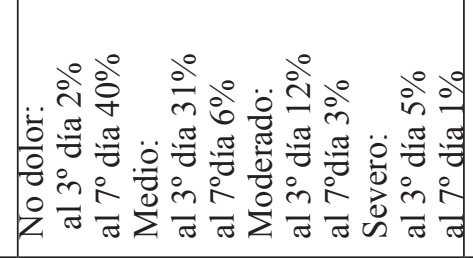 & \\
\hline 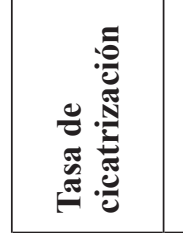 & & 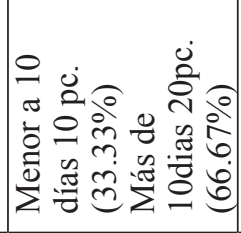 & & 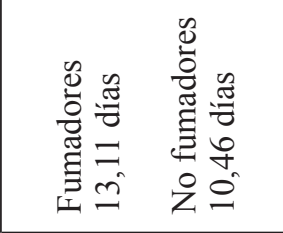 \\
\hline 䓀 & 旁 & 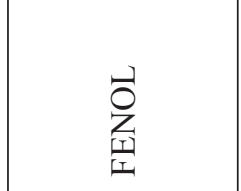 & 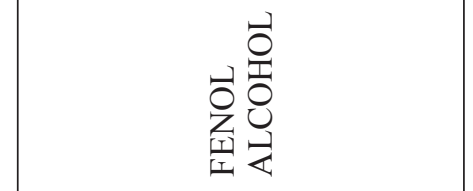 & 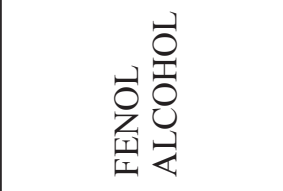 \\
\hline 离 & 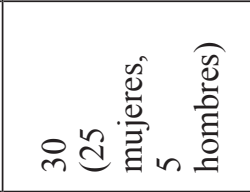 & 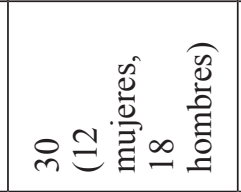 & in & 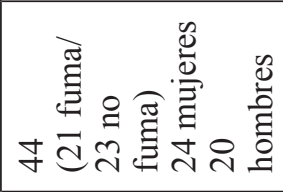 \\
\hline 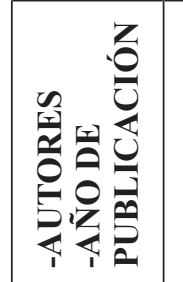 & 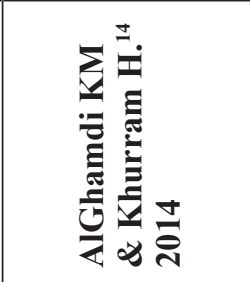 & 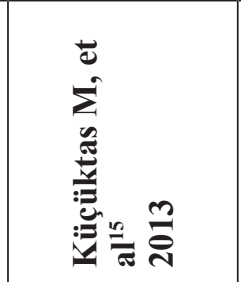 & 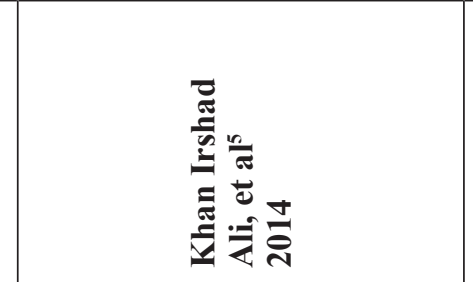 & 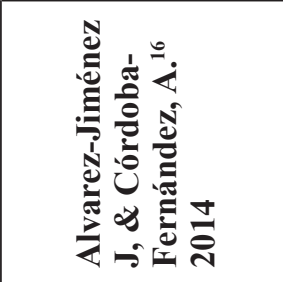 \\
\hline
\end{tabular}




\begin{tabular}{|c|c|c|c|c|c|}
\hline 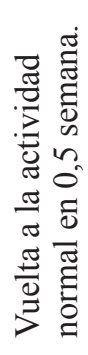 & & 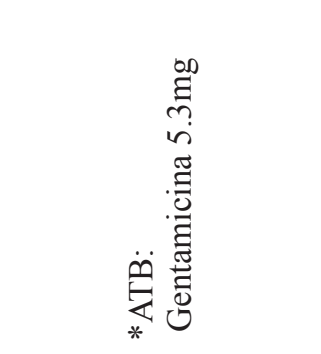 & 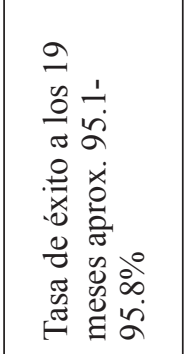 & 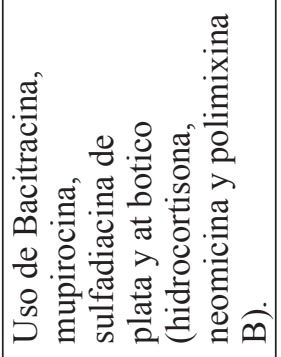 & \multirow{9}{*}{ 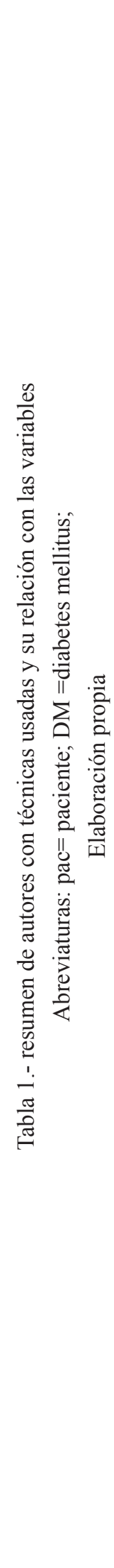 } \\
\hline \multirow{3}{*}{ 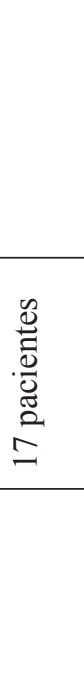 } & & 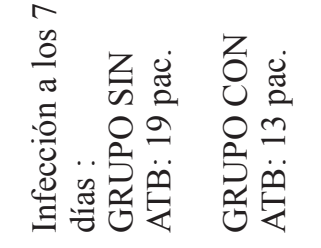 & & 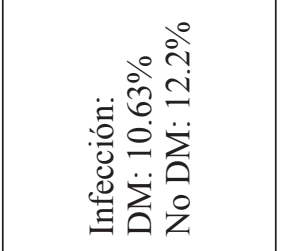 & \\
\hline & 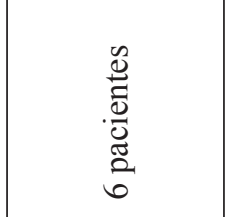 & 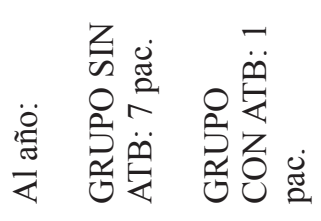 & & 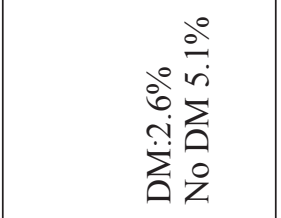 & \\
\hline & 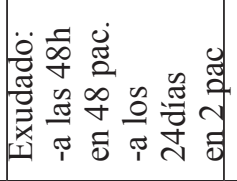 & & 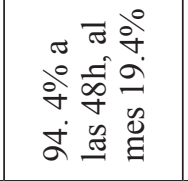 & & \\
\hline \multirow[t]{2}{*}{ 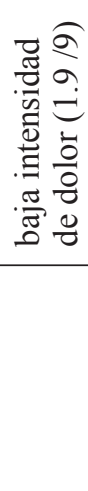 } & 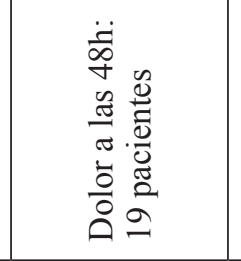 & & 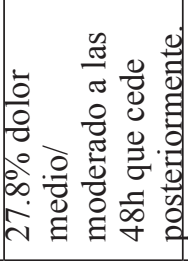 & & \\
\hline & 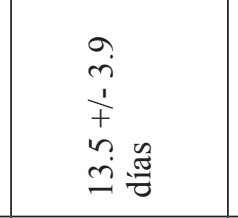 & & & 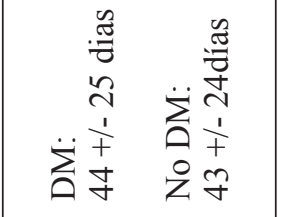 & \\
\hline 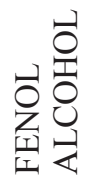 & 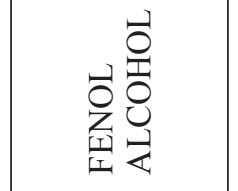 & 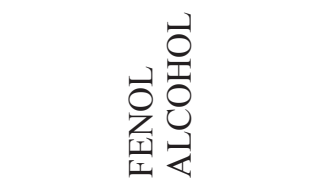 & \begin{tabular}{l}
$\overrightarrow{0}$ \\
$Z_{I}$ \\
\multicolumn{1}{|c}{}
\end{tabular} & 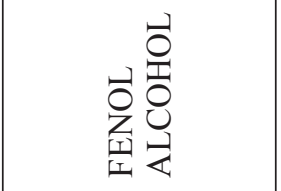 & \\
\hline$m$ & 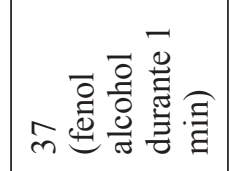 & 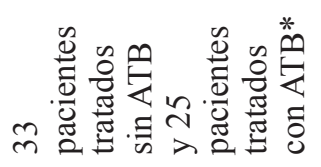 & 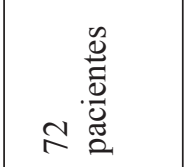 & 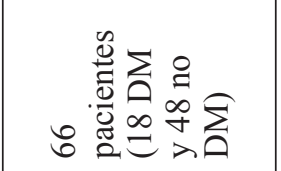 & \\
\hline 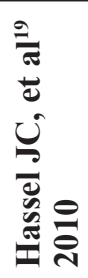 & 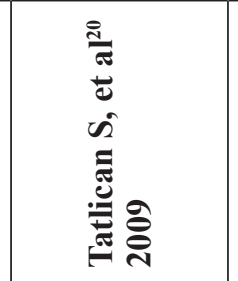 & 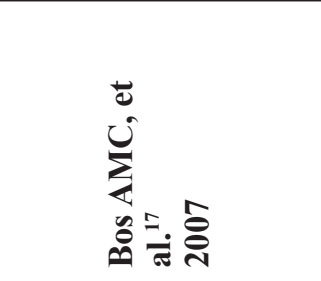 & 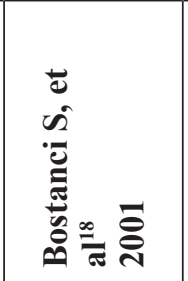 & 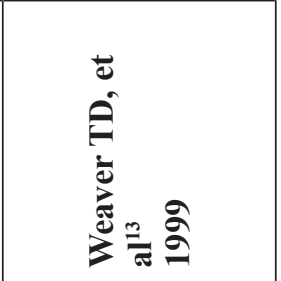 & \\
\hline
\end{tabular}




\section{Discusión}

La literatura consultada coincide en realizar matricectomía química con fenol alcohol frente a otros tipos de cirugías para el tratamiento de la onicocriptosis, siempre teniendo en cuenta la patología de cada paciente. Khan ${ }^{5}$, Joel ${ }^{11}$, Karaca $^{21}$, Zaraa ${ }^{22}$ y Cameron ${ }^{23}$ así lo exponen en sus estudios. En nuestra tabla se observa prevalencia de la técnica combinada fenol alcohol frente a fenol.

\subsection{Tiempo de cicatrización}

Con respecto al tiempo de cicatrización de la herida producida por la quemadura química generada por el fenol, Küçüktaş et al ${ }^{15}$ observaron que 10 de los 30 pacientes en los 10 primeros días tras la cirugía habían conseguido cicatrizar la herida, sin embargo, los 20 restantes tardaron más. AlvarezJiménez \& Córdoba-Fernández ${ }^{16}$ en su estudio comparativo de 2014 con pacientes fumadores y no fumadores, demostraron que los tiempos de cicatrización son mayores en pacientes fumadores debido a una menor concentración de oxígeno en sangre, disminución de la concentración de vitamina $\mathrm{C}$, alteración en el metabolismo del colágeno y afectación de la respuesta inflamatoria de las células; además concluyeron que retirar el tejido cauterizado tras la fenolización reduce el tiempo de cicatrización. Tatlican et $\mathrm{al}^{20}$ realizaron un estudio comparativo de los tiempos de aplicación del fenol durante 1-2-3 minutos y adujeron que el tiempo de cicatrización para el grupo en el que se utilizaba la aplicación del fenol durante un minuto era de $13.5+/-3.9$ días para cura completa de la herida, frente a tiempos más largos en los otros dos grupos. Sin embargo en el estudio de Weaver et $\mathrm{al}^{21}$ con pacientes diabéticos y no diabéticos, se observó que los tiempos de cicatrización son altos (43-44 días), sin apreciar diferencias entre ambos grupos.

\subsection{El dolor postquirúrgico}

AlGhamdi \& Khurram ${ }^{14}$ afirmaron que el dolor postquirúrgico se da en las primeras 30 horas; Khan, Irshad Ali, et $\mathrm{al}^{5}$ lo expusieron mediante una escala de dolor ( no dolor, medio, moderado y severo), y observaron que el mayor pico de pacientes con dolor se da al tercer día y es dolor medio. Hassel eta ${ }^{19}$ demostraron la intensidad baja de dolor de 1,9 sobre 10 con la técnica de fenol, mediante una escala de dolor graduada y validada. Tatlican et $\mathrm{al}^{20}$ hicieron referencia de que a las $48 \mathrm{~h}$ fueron 19 pacientes de los 37 que tuvieron dolor en el grupo en el que se usó el fenol durante 1 minuto, sin embargo no encontraron diferencias significativas en los grupos de fenol durante 2 minutos y 3 minutos. Bostanci et $\mathrm{al}^{18}$ recogieron un $27.8 \%$ de los pacientes con dolor medio/moderado a las $48 \mathrm{~h}$ que cedió posteriormente.

\subsection{El exudado de la herida postquirúrgica}

AlGhamdi \& Khurram ${ }^{14}$ mostraron que en las primeras $26.43 \mathrm{~h}$ apareció exudado de la herida, sin embargo en el estudio de Tatlican et $\mathrm{al}^{20}$ el exudado se dio a las $48 \mathrm{~h}$ en 48 de los 37 pacientes que intervinieron, $y$ fue disminuyendo progresivamente, al igual que en el estudio de Bostanci et $\mathrm{al}^{18}$ en el que se observó un mayor número de pacientes con exudado en las primeras $48 \mathrm{~h}$ postquirúrgicas (94.4\%).

Cabe destacar entre la bibliografía consultada que en el estudio de Hassel et $\mathrm{al}^{19}$, de los 33 pacientes tratados con fenol se observó que en 17 existía recurrencia de la onicocriptosis, de los cuales 12 se manifestaron en nuevos episodios de onicocriptosis y 5 en formación de espículas de inclusión, siendo el tiempo de aparición de $9.9+/-5.4$ meses.

\subsection{Infección}

En cuanto a la variable de infección, cabe destacar que la administración de profilaxis antibiótica en los pacientes sin riesgo, previo a la cirugía y como tratamiento protololarizado postquirúrgico, podría enmascarar signos de infección, así como mejorar el tiempo de cicatrización de la herida y los datos obtenidos podrían estar alterados ${ }^{24}$.

Dentro de la técnica fenol alcohol, hemos encontrado variantes a la hora de realizar lavado entre aplicaciones de fenol, si bien en lugar de utilizar alcohol con el objetivo de neutralizar la acción del fenol, estudios como el de Cordoba Diaz, Damian et $\mathrm{al}^{25}$ demostraron que el uso de una solución de poliexamida $0.1 \%$ elimina eficazmente el fenol sobrante de la herida quirúrgica en relación a la irrigación con alcohol (57.74\% alcohol Vs $75.74 \%$ poliexamida $0.1 \%$ ). Estos autores, en 2013, realizaron un estudio en el que 
añadían la clorhexidina al $0.5 \%$ al alcohol para eliminar el exceso de fenol ${ }^{26}$, y demostraron la efectividad si solo se realizaba con una única irrigación del área intervenida ${ }^{27}$.

\section{Conclusiones}

De los estudios revisados, se puede apreciar prevalencia en el uso de fenol alcohol frente a fenol para el tratamiento de la onicocriptosis, con sus variantes a la hora de realizar la técnica. El tiempo de cicatrización puede verse alterado por diferentes factores tales como el tabaco, el uso de antibióticos o enfermedades concomitantes, así como el exudado de la herida, que además depende del tiempo de aplicación del fenol que hayamos llevado a cabo. El dolor postquirúrgico podemos señalar que en general es de media/ baja intensidad y que va cediendo gradualmente a partir de las $48 \mathrm{~h}$ de la intervención. Cabe destacar entre la bibliografía consultada que solo el estudio de Hassel et al ${ }^{19}$ mostraba datos significativos de alta recurrencia tras la cirugía. En cuanto a la variable de infección, puede estar sesgada por el uso de antibióticos y no es valorable.

\section{Bibliografía}

1. Martinez-Nova A, Sanchez-Rodriguez R, Alonso-Pena D. A new onychocryptosis classification and treatment plan. J Am Podiatr Med Assoc 2007;97(5):389-393.

2. Bean WB. Nail growth: a twenty-year study. Arch Intern Med 1963;111(4):476-482.

3. Nova AM. Podología: atlas de cirugía ungueal. : Ed. Médica Panamericana; 2006.

4. Pearson HJ, Bury RN, Wapples J, Watkin DF. Ingrowing toenails: is there a nail abnormality? A prospective study. J Bone Joint Surg Br 1987 Nov;69(5):840-842.

5. Khan IA, Shah SF, Waqar SH, Abdullah MT, Malik Z, Zahid MA. Treatemnt of ingrown toe nail comparison of phenolization after partial nail avulsion and partial nail avulsion alone. J Ayub Med Coll Abbottabad 2014;26(4):522-525.

6. Bryant A, Knox A. Ingrown toenails: the role of the GP. Aust Fam Physician 2015;44(3):102.

7. Gavillero A, Arxé D, de Veciana EG, Ogalla JM, de Planell E, Zalacain A, et al. Estudio estadístico en cirugía ungueal. El Peu 2005;25(1):20-30.

8. Carmona FJG, Morato DF. Tratamiento quirúrgico de la onicocriptosis. : Aula Médica; 2003.

9. Connelley LK, Jr, Dinehart SM, McDonald R. Onychocryptosis associated with the treatment of onychomycosis. J Am Podiatr Med Assoc 1999 Aug; 89(8):424-426.

10. Weaver TD, Jespersen DL. Multiple onychocryptosis following treatment of onychomycosis with oral terbinafine. Cutis 2000 Sep; 66(3):211-212.

11. Joel J, Heidelbaugh A, Hobart L. Management of The Ingrown Toenail. American Family Phys 2009; 79: 303 2009; 8.

12. Mozena JD. The Mozena Classification System and treatment algorithm for ingrown hallux nails. J Am Podiatr Med Assoc 2002; 92(3):131-135.

13. Felton PM, Weaver TD. Phenol and alcohol chemical matrixectomy in diabetic versus nondiabetic patients. A retrospective study. J Am Podiatr Med Assoc 1999 Aug;89(8):410-412.

14. AlGhamdi K, Khurram H. Nail Tube Splinting Method Versus Lateral Nail Avulsion With Phenol Matricectomy. Dermatologic Surgery. 2014;40(11):1214-1220.

15. Küçüktaş M, Kutlubay Z, Yardimci G, Khatib R, Tüzün Y. Comparison of Effectiveness of Electrocautery and Cryotherapy in Partial Matrixectomy After Partial Nail Extraction in the Treatment of Ingrown Nails. Dermatologic Surgery. 2013;39(2):274-280.

16. Alvarez-Jiménez J, Córdoba-Fernández A. Influence of Smoking on Wound Healing in Patients Undergoing Nail Matrix Phenolization. Advances in Skin \& Wound Care. 2014;27(5):229-236.

17. Bos A, van Tilburg M, van Sorge A, Klinkenbijl J. Randomized clinical trial of surgical technique and local antibiotics for ingrowing toenail. British Journal of Surgery. 2007;94(3):292-296.

18. Bostanci, Pelin Ekmekçi, Er S. Chemical Matricectomy with Phenol for the Treatment of Ingrowing Toenail: A Review of the Literature and Follow-up of 172 Treated Patients. Acta Dermato-Venereologica. 2001;81(3):181-183.

19. Hassel J, Hassel A, Loeser C. Phenol Chemical Matricectomy Is Less Painful, with Shorter Recovery Times but Higher Recurrence Rates, Than Surgical Matricectomy: A Patient's View. Dermatologic Surgery. 2010;36(8):1294-1299. 
20. Tatlican S., Yamangokturk B, Eren C, Eskioglu F, Adiyaman S. Comparison of phenol applications of different durations for the cauterization of the germinal matrix: an efficacy and safety study. Acta Orthopaedica et Traumatologica Turcica. 2009;43(4):298-302.

21. Karaca N, Dereli T. Treatment of Ingrown Toenail With Proximolateral Matrix Partial Excision and Matrix Phenolization. The Annals of Family Medicine. 2012;10(6):556-559

22. Zaraa I, Dorbani I, Hawilo A, Mokni M, Ben Osman A. Segmental phenolization for the treatment of ingrown toenails: technique report, follow up of 146 patients, and review of the literature. Dermatology online journal 2013; 19(6).

23. Cameron PF. Ingrowing toenails: an evaluation of two treatments. Br Med J (Clin Res Ed) 1981 Sep 26;283(6295):821-822.

24. Córdoba-Fernández A, Ruiz-Garrido G, Canca-Cabrera Á. Algorithm for the management of antibiotic prophylaxis in onychocryptosis surgery. The Foot. 2010;20(4):140-145.

25. Cordoba Diaz D, Becerro de Bengoa Vallejo R, Losa Iglesias M, Cordoba Diaz M. Polihexanide solution is more efficient than alcohol to remove phenol in chemical matricectomy: an in vitro study. Dermatologic Therapy. 2014;27(6):369-372.

26. Diaz D, de Bengoa Vallejo R, Iglesias M, Diaz M. Alcohol Plus Chlorhexidine is More Efficient Than Alcohol Alone for Phenol-Based Chemical Matricectomy: An In Vitro Study. Dermatologic Surgery. 2013;39(9):1363-1367.

27. Cordoba-Diaz D, Becerro de Bengoa Vallejo R, Losa Iglesias M, Cordoba-Díaz M. Effectiveness of standard lavage with supplemental chlorhexidine in patients undergoing chemical matricectomy for ingrown toenails: A clinical trial. Journal of the American Academy of Dermatology. 2014;70(6):10921095. 